\title{
A SYSTEMATIC REVIEW AND SINGLE-CENTRE EXPERIENCE OF URETERORENOSCOPY UNDER LOCAL ANESTHETIC: A SAFER OPTION FOR ANESTHETICALLY HIGH-RISK PATIENTS?
}

Ali Tasleem ${ }^{1}$, Sachin Yallappa ${ }^{2}$, Michael Mikhail, ${ }^{1}$ Tarik Amer $^{2}$, Peter Pietrzak ${ }^{1}$, Peter Acher ${ }^{1}$, Antony Young ${ }^{1}$

${ }^{1}$ Southend University Hospital NHS Foundation Trust, Urology, Westcliff-on-Sea, Essex, UK

${ }^{2}$ Queen Elizabeth University Hospital, Urology Department. Glasgow Urological Research Unit, Department of Urology, Glasgow, Scotland, UK

Corresponding Author: amt03@doctors.org.uk

Submitted: March 10, 2019. Accepted: April 14, 2019. Published: May 1, 2019.

\begin{abstract}
Patients are living longer with an increasing number of co-morbidities. Minimally invasive ureterorenoscopy (URS) to manage upper tract calculi or transitional cell carcinoma (TCC) can be performed under general or spinal anesthesia; however, certain co-morbid patients are not suitable for this and may benefit from a different approach. We report on URS under local anesthesia (LA) using intra-ureteric marcaine as the primary form of anesthesia. We also aimed to perform a robust systematic review of this topic.

A retrospective analysis over 6 years was undertaken on all patients who underwent URS for calculi or TCC under LA, with the use of intra-urethral lidocaine gel (2\%) and intra-ureteric marcaine $(0.5 \%, 20 \mathrm{~mL})$ with sedoanalgesia as an adjunct. A systematic review and all English language articles on ureteroscopic procedures with the use of LA with or without intravenous sedoanalgesia were selected and data extracted.

In our case series, 12 patients had a total of 42 procedures. Stone size varied from 4-35 mm. Twenty-two percent of procedures (9/41) did not require any sedation or intravenous analgesia as an adjunct to the bupivacaine with a further $49 \%$ (20/41) requiring midazolam. (The anesthetic chart was not available for one procedure.) No procedures were abandoned and there were no conversions to general $/$ spinal anesthesia. There were no complications secondary to the use of LA. Eighty-one percent of cases (34/42) were performed as a day-case or overnight stays. The complication rate was similar to that for conventional anesthesia. The systematic review yielded 1121 procedures from 11 papers and 7 countries. In 32 cases the procedure was converted to general anesthesia. Stone clearance rates were between $78-100 \%$. The procedures were well tolerated in $80-90 \%$ of cases.

This study highlights that URS can be safely performed under LA. It is well tolerated and represents an option for carefully selected patients who have been adequately counselled, and who would be at high risk from anesthesia. Such patients may otherwise be considered "unfit" for endourological intervention.
\end{abstract}

Keywords: ureteric stones, ureteroscopy, laser, renal stone 
In 1912, Hugh Hampton Young accidentally entered the dilated ureter of a child with posterior urethral valves thereby performing the first diagnostic ureteroscopy. This was published years later in 1929. ${ }^{1}$ In 1977, Goodman first reported the use of an 11Fr pediatric cystoscope in adult women for ureteric pathology. ${ }^{2}$ A few years later in 1980, the first ureteroscope that could reach the renal pelvis was introduced. Over the decades, ureteroscopy has become ubiquitous with the miniaturization and improved optics of semi-rigid and flexible ureterorenoscopes. These procedures have become mainstream modalities for the management of urolithiasis and increasingly upper tract malignancies. ${ }^{3}$ Although many institutions perform ureterorenscopy on a day-case basis, some co-morbid patients are not surgical candidates for ureteroscopic procedures under general or spinal anesthetic. The intent of this article is therefore twofold: (1) to report on the viability of performing ureteroscopy under local anesthetic (LA) in 12 of our patients over 6 years and (2) perform a robust systematic review of this topic.

\section{METHODS FOR SYSTEMATIC REVIEW OF THE LITERATURE}

Three of the authors (AT, SY, and TA) performed a literature search using the key words: ureteroscopy, stones, local anesthesia, regional anesthesia, flexible ureteroscopy and rigid ureteroscopy. Google Scholar, PubMed, EMBASE and Cochrane databases were searched. English language articles on ureteroscopic procedures with the use of LA with or without intravenous analgesia and/or sedation were selected. Reference lists of articles were also assessed to ensure all relevant articles were included. Where full articles were not available, abstracts were utilized if sufficient data were extractable.

Our outcome measures were: (1) Major and minor complications, (2) Stone clearance rates, (3) Patient tolerability, and (4) Length of hospital stay.

\section{CASE SERIES METHODOLOGY AND PATIENT SELECTION}

A retrospective analysis was made of all patients who underwent ureteroscopy/flexible ureterorenoscopy (URS/FURS) for calculi or upper tract transitional cell carcinoma (TCC) under LA with the use of intra-ureteric marcaine $(0.5 \%, 20 \mathrm{~mL})$. Marcaine was instilled under pressure into the ureter using a $5 \mathrm{Fr}$ ureteric catheter prior to commencing URS.

\section{PATIENT SELECTION}

We selected adult patients who required URS and laser fragmentation for stone disease or for TCC in whom general or spinal anesthesia was high risk or contraindicated for a variety of reasons: high body-mass index, difficult airway, asbestosis-related pulmonary fibrosis, previous severe postoperative cardiorespiratory failure, and previous difficulties with intubation. Patients who expressed a desire to avoid general or spinal anesthetic were provided with the option of a procedure under sedoanalgesia with LA. Where appropriate, extra-corporeal shockwave lithotripsy (ESWL) was attempted in the first instance to avoid ureteroscopy, but was unsuccessful.

\section{PROCEDURE}

All procedures were performed in the hospital operating room. An anesthetist was involved in all cases to administer sedation, provide intravenous analgesia when required, and for patient monitoring. Patients would receive sedation and intravenous analgesia in the anesthetic room. The nature of the sedation and analgesia was determined on a case-by-case basis and by each individual anesthetist's preferences and patient tolerability (Tables 1 and 2). Prophylactic intravenous antibiotics were administered prior to commencing endoscopy. Once in theatre, patients were positioned in the lithotomy position and prepared and draped as for cystoscopy.

Intra-urethral lidocaine gel $\left(2 \%, 11 \mathrm{~mL}\right.$ Instillagel $\left.{ }^{\circledR}\right)$ was instilled prior to performing cystoscopy using a 17 Fr Olympus ${ }^{\circledR}$ rigid cystoscope. A Sensor ${ }^{\circledR}$ guide wire (Boston Scientific $\AA$ ) was used to cannulate the ureteric orifice and the wire passed to the kidney under image intensifier guidance followed by a duallumen catheter over this. Through this $20 \mathrm{~mL} 0.5 \%$ marcaine was infused at pressure resulting in a degree of extravasation.

Following a delay of 3-5 minutes and with close attention to patient behaviour, a graduated $6.5 / 7.5 \mathrm{Fr}$ Wolf $\AA$ semi-rigid ureteroscope was inserted alongside the safety Sensor ${ }^{\circledR}$ wire for ureteric pathology. For

J Endolum Endourol Vol 2(2):e1-e14; May 1, 2019.

This article is distributed under the terms of the Creative Commons Attribution-Non

Commercial 4.0 International License. (T) Tasleem et al. 


\begin{tabular}{|c|c|c|c|c|}
\hline $\begin{array}{l}\overrightarrow{0} \\
\stackrel{\circ}{0} \\
0\end{array}$ & 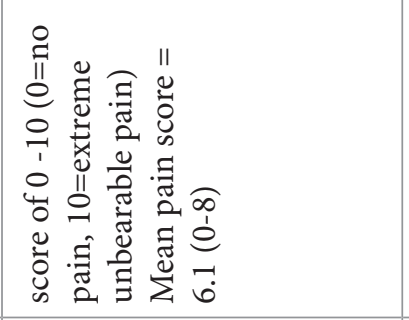 & 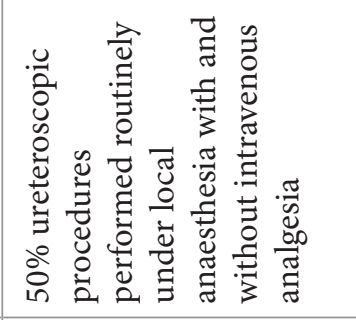 & 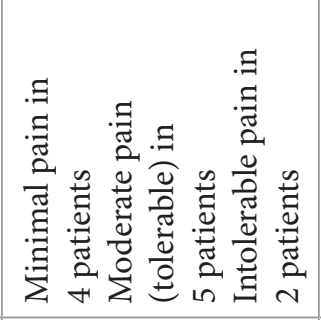 & 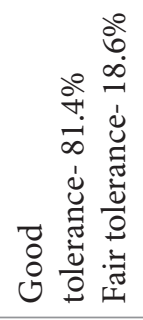 \\
\hline 芌 & 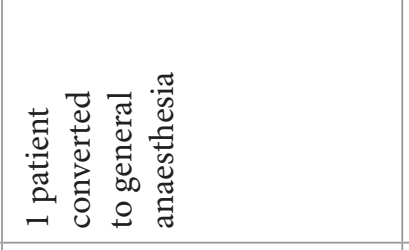 & 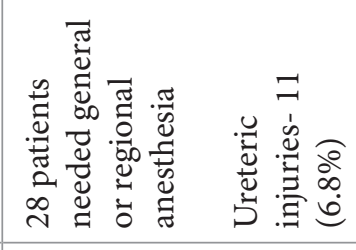 & 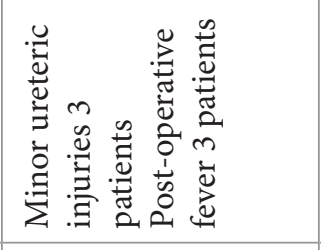 & 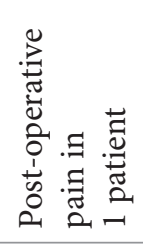 \\
\hline @े & $\stackrel{\circ}{\circ}$ & $\not \triangleleft$ & 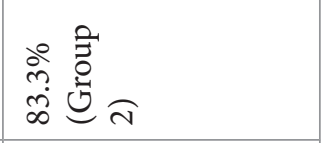 & ถั̊ \\
\hline in & $\overrightarrow{0}$ & 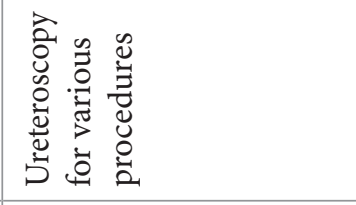 & §ి & $\ddot{b}$ \\
\hline 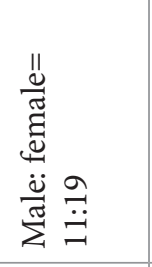 & 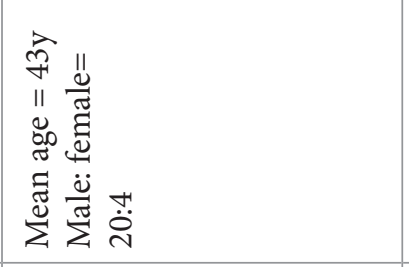 & 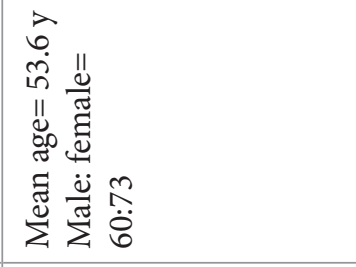 & 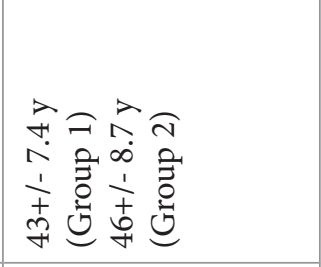 & \\
\hline 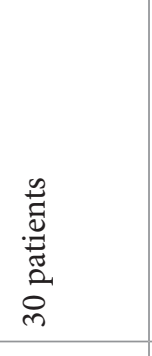 & 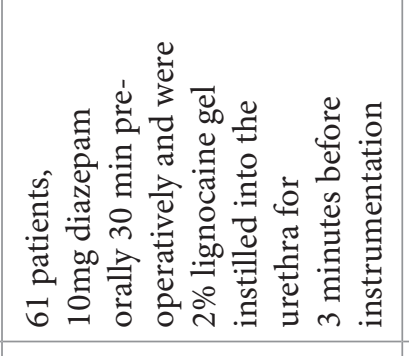 & 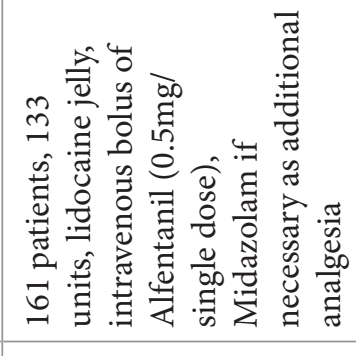 & 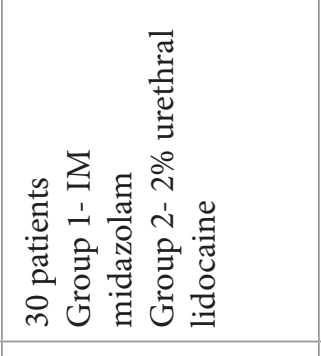 & 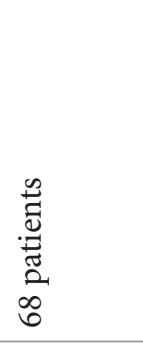 \\
\hline 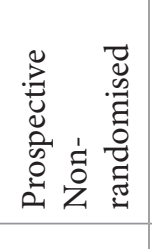 & 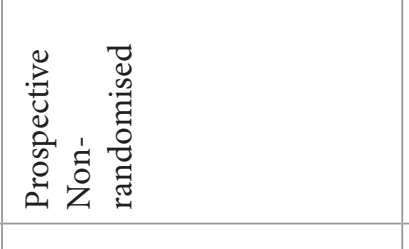 & 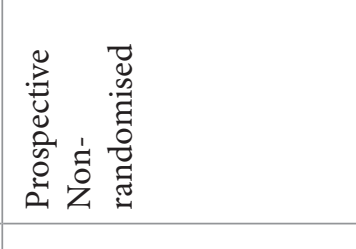 & 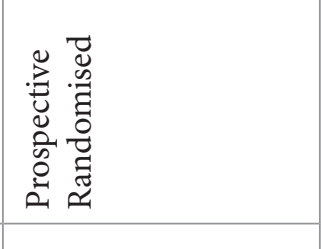 & 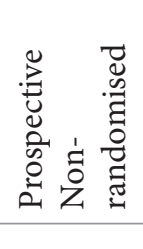 \\
\hline 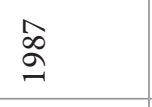 & \& & $\stackrel{2}{2}$ & 点 & $\stackrel{2}{\circ}$ \\
\hline 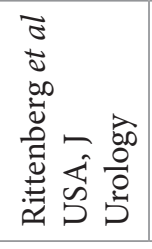 & 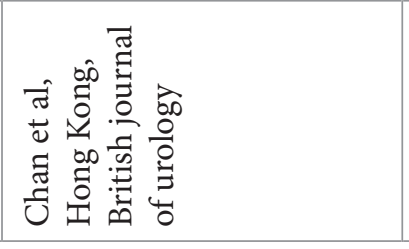 & 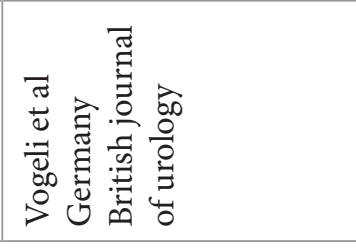 & 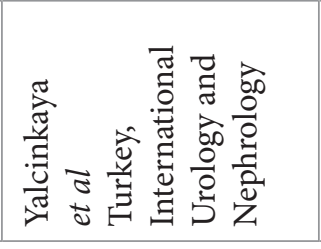 & 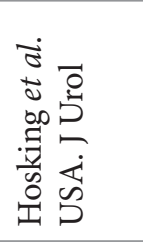 \\
\hline
\end{tabular}

J Endolum Endourol Vol 2(2):e1-e14; May 1, 2019.

This article is distributed under the terms of the Creative Commons Attribution-Non Commercial 4.0 International License. OTasleem et al. 


\begin{tabular}{|c|c|c|c|c|}
\hline & 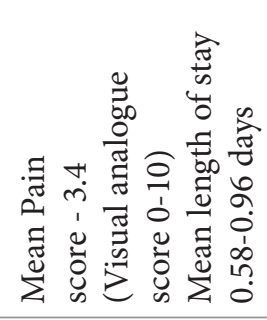 & 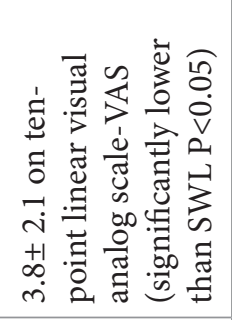 & 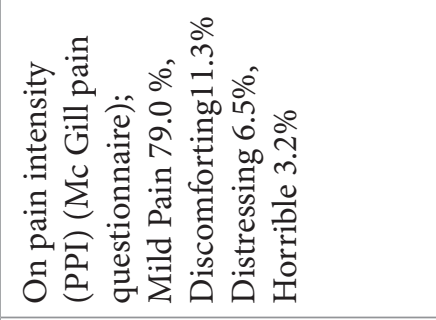 & \\
\hline 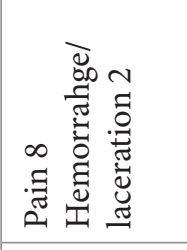 & 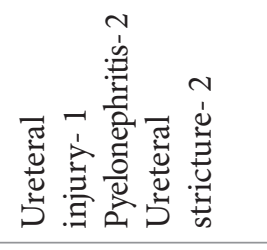 & & 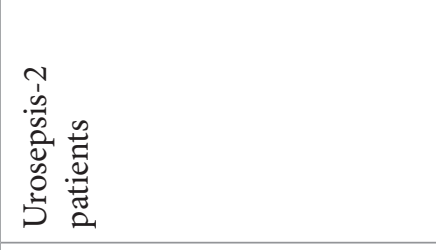 & 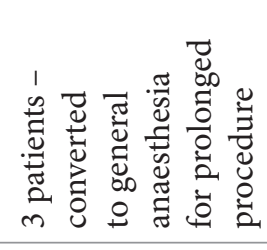 \\
\hline$\underset{\infty}{\stackrel{\circ}{ }}$ & ळे & & 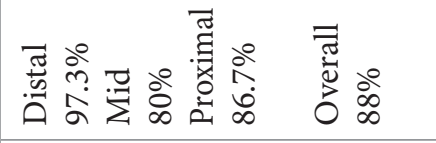 & ふे \\
\hline$\ddot{m}$ & ષ્సి & & $\stackrel{\sharp}{\Xi}$ & $\stackrel{\sim}{\sim}$ \\
\hline & 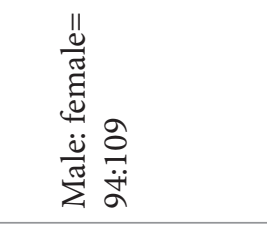 & 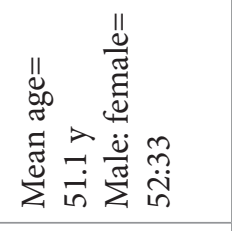 & 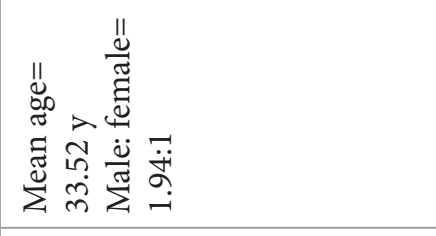 & 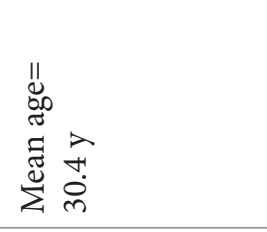 \\
\hline 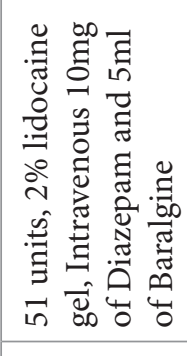 & 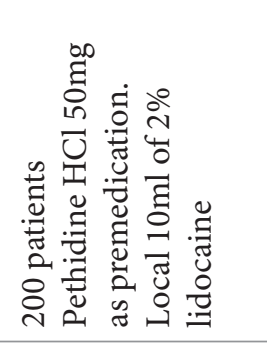 & 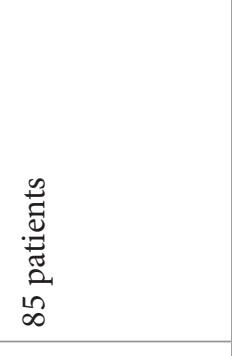 & 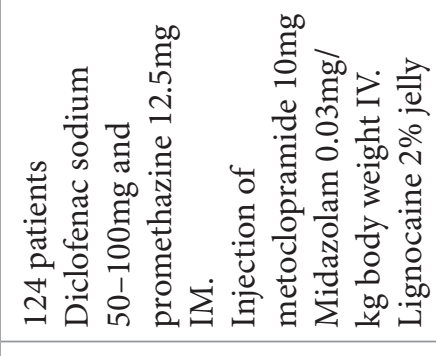 & 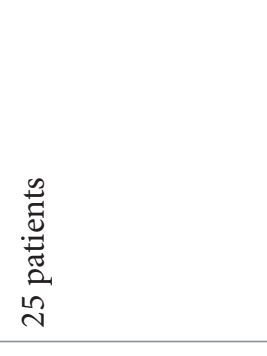 \\
\hline 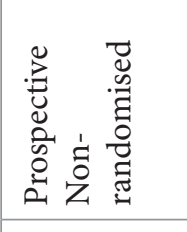 & 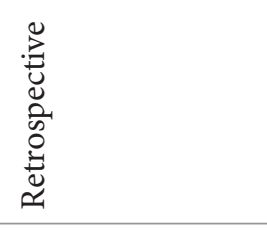 & 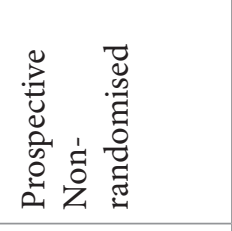 & 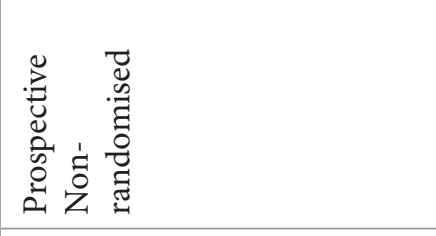 & 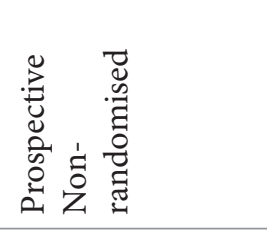 \\
\hline बूे & 容 & ڤ్ & ڤి & ๕্ণ \\
\hline 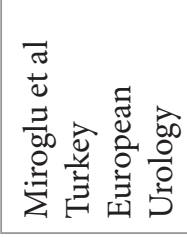 & 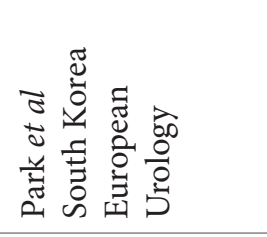 & 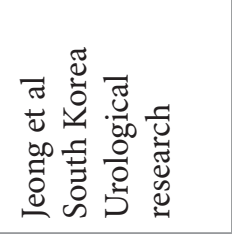 & 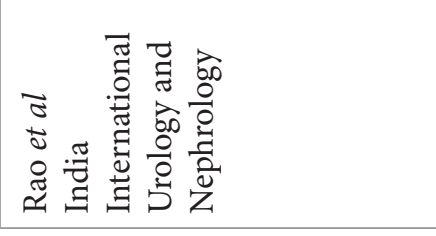 & 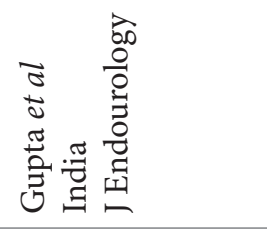 \\
\hline
\end{tabular}

J Endolum Endourol Vol 2(2):e1-e14; May 1, 2019.

This article is distributed under the terms of the Creative Commons Attribution-Non Commercial 4.0 International License. OTasleem et al. 


\begin{tabular}{|c|c|}
\hline 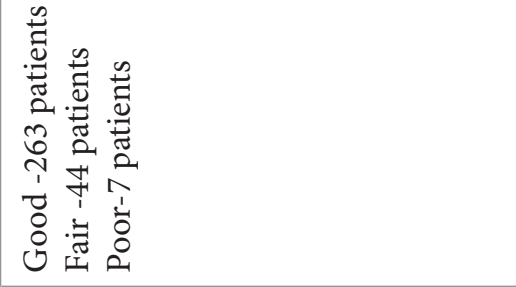 & 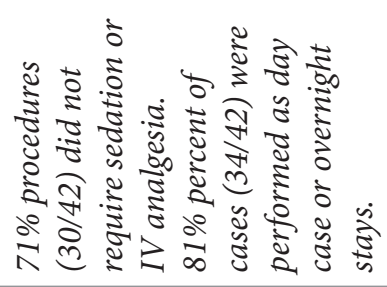 \\
\hline 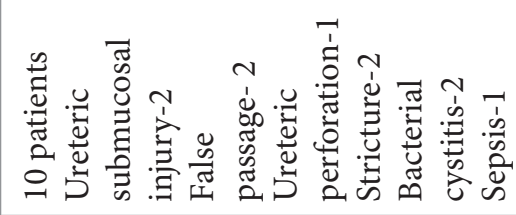 & 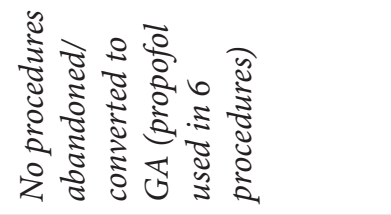 \\
\hline 웅 & 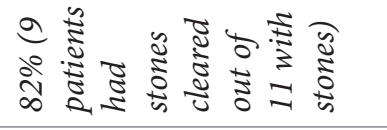 \\
\hline$\frac{\vec{m}}{m}$ & 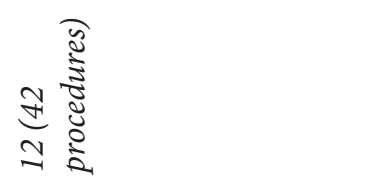 \\
\hline 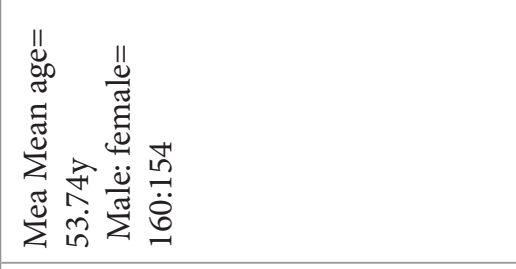 & 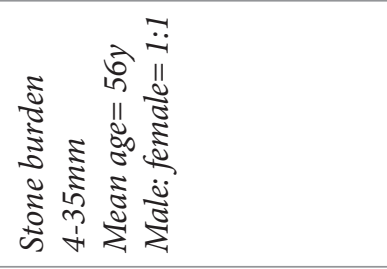 \\
\hline 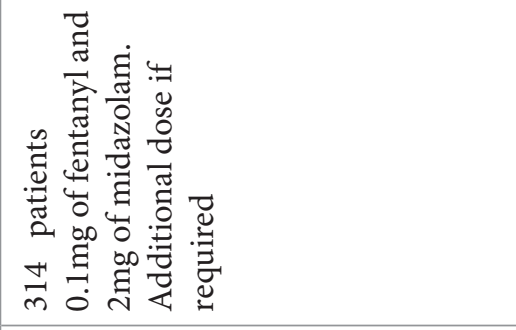 & 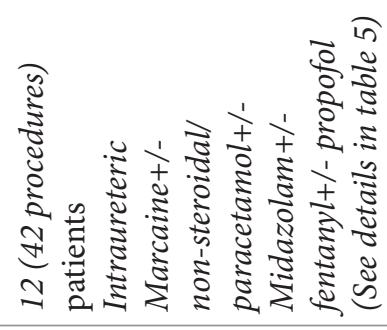 \\
\hline 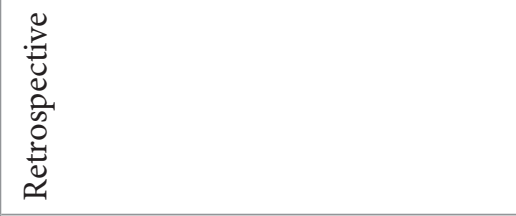 & 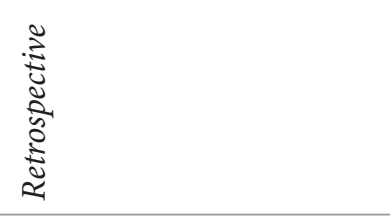 \\
\hline$\stackrel{0}{\stackrel{2}{\sim}}$ & $\widehat{\stackrel{\sim}{i}}$ \\
\hline 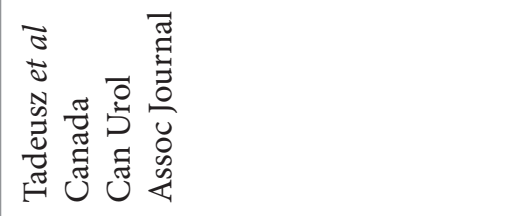 & 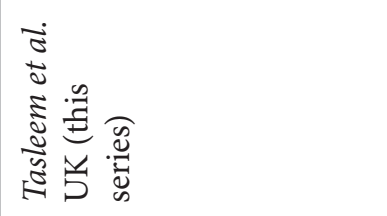 \\
\hline
\end{tabular}

J Endolum Endourol Vol 2(2):e1-e14; May 1, 2019.

This article is distributed under the terms of the Creative Commons Attribution-Non Commercial 4.0 International License. OTasleem et al. 
TABLE 2 Current Case Series

\begin{tabular}{|c|c|c|c|c|c|c|}
\hline Case & Pathology & Site & Size & Scope used & Sedoanalgesia & Indication for LA \\
\hline $\begin{array}{l}\text { Case } 1 \\
\text { Procedure } 1\end{array}$ & Stone & Distal ureter & $5 \mathrm{~mm}$ & DUR-D & $\begin{array}{l}\text { Paracetamol, } \\
\text { diclofenac, } \\
\text { midazolam, } \\
\text { intrauretric marcaine }\end{array}$ & \multirow{2}{*}{$\begin{array}{l}\text { ESWL- stone } \\
\text { not visualised. } \\
\text { Failed intubation- } \\
\text { laryngospasm. } \\
\text { Failed spinal } \\
\text { anesthetic. }\end{array}$} \\
\hline $\begin{array}{l}\text { Case } 1 \\
\text { Procedure } 2\end{array}$ & Stone & Distal ureter & $4 \mathrm{~mm}$ & URF-V & $\begin{array}{l}\text { Paracetamol, } \\
\text { diclofenac, } \\
\text { midazolam, } \\
\text { intrauretric marcaine }\end{array}$ & \\
\hline $\begin{array}{l}\text { Case } 2 \\
\text { Procedure } 1\end{array}$ & Stone & $\begin{array}{l}\text { Renal pelvis } \\
\text { and }+ \text { lower } \\
\text { pole }\end{array}$ & $\begin{array}{l}\text { Up to } 33 \\
\mathrm{~mm}\end{array}$ & URF-V & $\begin{array}{l}\text { Paracetamol, } \\
\text { diclofenac, midazolam, } \\
\text { intrauretric marcaine, } \\
\text { fentanyl }\end{array}$ & \multirow[t]{4}{*}{ Patient choice } \\
\hline $\begin{array}{l}\text { Case } 2 \\
\text { Procedure } 2\end{array}$ & Stone & $\begin{array}{l}\text { Multiple: } \\
\text { Lower pole }\end{array}$ & $8-10 \mathrm{~mm}$ & URF-P5 & $\begin{array}{l}\text { Paracetamol, } \\
\text { diclofenac, midazolam, } \\
\text { intrauretric marcaine }\end{array}$ & \\
\hline $\begin{array}{l}\text { Case } 2 \\
\text { Procedure } 3\end{array}$ & Stone & $\begin{array}{l}\text { Multiple: } \\
\text { Lower pole }\end{array}$ & $10-15 \mathrm{~mm}$ & & $\begin{array}{l}\text { Paracetamol, } \\
\text { diclofenac, midazolam, } \\
\text { intrauretric marcaine }\end{array}$ & \\
\hline $\begin{array}{l}\text { Case } 2 \\
\text { Procedure } 4\end{array}$ & Stone & Lower pole & & & $\begin{array}{l}\text { Paracetamol, } \\
\text { diclofenac, midazolam, } \\
\text { intrauretric marcaine }\end{array}$ & \\
\hline $\begin{array}{l}\text { Case } 3 \\
\text { Procedure } 1\end{array}$ & TCC & Distal ureter & & DUR-D & $\begin{array}{l}\text { Paracetamol, } \\
\text { diclofenac, } \\
\text { midazolam, fentanyl, } \\
\text { propofol }\end{array}$ & $\begin{array}{l}\text { Severe chronic } \\
\text { obstructive } \\
\text { pulmonary disease, } \\
\text { obstructive sleep } \\
\text { apnea Refused } \\
\text { spinal. }\end{array}$ \\
\hline $\begin{array}{l}\text { Case } 4 \\
\text { Procedure } 1\end{array}$ & Stone & $\begin{array}{l}\text { Upper ureter } \\
\text { Distal ureter }\end{array}$ & $\begin{array}{l}10 \mathrm{~mm} \\
4 \mathrm{~mm}\end{array}$ & URF-P5 & $\begin{array}{l}\text { Paracetamol, } \\
\text { diclofenac }\end{array}$ & Morbid obesity. \\
\hline $\begin{array}{l}\text { Case } 5 \\
\text { Procedure } 1\end{array}$ & Stone & Renal pelvis & $30 \mathrm{~mm}$ & & $\begin{array}{l}\text { Paracetamol, } \\
\text { midazolam, } \\
\text { intrauretric marcaine }\end{array}$ & \multirow{4}{*}{$\begin{array}{l}\text { Failed ESWL. } \\
\text { Ischaemic heart } \\
\text { disease, chronic } \\
\text { obstructive } \\
\text { pulmonary disease, } \\
\text { kyphoscoliosis, } \\
\text { obesity, difficult } \\
\text { extubation in past. }\end{array}$} \\
\hline $\begin{array}{l}\text { Case } 5 \\
\text { Procedure } 2\end{array}$ & Stone & $\begin{array}{l}\text { Lower pole } \\
\text { Renal pelvis }\end{array}$ & $7-8 \mathrm{~mm}$ & DUR-D & $\begin{array}{l}\text { Paracetamol, } \\
\text { midazolam, } \\
\text { intrauretric marcaine }\end{array}$ & \\
\hline $\begin{array}{l}\text { Case } 5 \\
\text { Procedure } 3\end{array}$ & Stone & $\begin{array}{l}\text { Upper, middle } \\
\text { and distal } \\
\text { ureter }\end{array}$ & & URF-V & $\begin{array}{l}\text { Paracetamol, } \\
\text { midazolam, fentanyl, } \\
\text { propofol, intrauretric } \\
\text { marcaine }\end{array}$ & \\
\hline $\begin{array}{l}\text { Case } 5 \\
\text { Procedure } 4\end{array}$ & Stone & $\begin{array}{l}\text { Multiple lower } \\
\text { pole }\end{array}$ & & DUR-D & & \\
\hline
\end{tabular}


TABLE 2 Current Case Series (Continued)

\begin{tabular}{|c|c|c|c|c|c|c|}
\hline $\begin{array}{l}\text { Case } 6 \\
\text { Procedure } 1\end{array}$ & Stone & $\begin{array}{l}\text { PUJ and lower } \\
\text { pole }\end{array}$ & $4-6 \mathrm{~mm}$ & & $\begin{array}{l}\text { Paracetamol, } \\
\text { midazolam, fentanyl, } \\
\text { propofol }\end{array}$ & $\begin{array}{l}\text { Failed ESWL. } \\
\text { Obesity, limited } \\
\text { neck extension and } \\
\text { mouth opening }\end{array}$ \\
\hline $\begin{array}{l}\text { Case } 7 \\
\text { Procedure } 1\end{array}$ & Stone & Renal pelvis & $10-20 \mathrm{~mm}$ & & $\begin{array}{l}\text { Paracetamol, } \\
\text { midazolam, } \\
\text { intrauretric marcaine, }\end{array}$ & \multirow{9}{*}{$\begin{array}{l}\text { Previous prolonged } \\
\text { intensive case } \\
\text { unit admission } \\
\text { following GA } \\
\text { for percutaneous } \\
\text { nephrolithotomy }\end{array}$} \\
\hline $\begin{array}{l}\text { Case } 7 \\
\text { Procedure } 2\end{array}$ & Stone & Renal pelvis & & & $\begin{array}{l}\text { Paracetamol, } \\
\text { intrauretric marcaine, }\end{array}$ & \\
\hline $\begin{array}{l}\text { Case } 7 \\
\text { Procedure } 3\end{array}$ & Stone & $\begin{array}{l}\text { Lower and } \\
\text { mid poles and } \\
\text { renal pelvis }\end{array}$ & & & $\begin{array}{l}\text { Paracetamol, } \\
\text { intrauretric marcaine, }\end{array}$ & \\
\hline $\begin{array}{l}\text { Case } 7 \\
\text { Procedure } 4\end{array}$ & Stone & $\begin{array}{l}\text { Bilateral renal } \\
\text { pelvis and left } \\
\text { ureter }\end{array}$ & $\begin{array}{l}\text { Staghorn } \\
\text { (Right) }\end{array}$ & & $\begin{array}{l}\text { Paracetamol, } \\
\text { midazolam, } \\
\text { intrauretric marcaine, }\end{array}$ & \\
\hline $\begin{array}{l}\text { Case } 7 \\
\text { Procedure } 5\end{array}$ & Stone & Renal pelvis & & & $\begin{array}{l}\text { Paracetamol, } \\
\text { midazolam, } \\
\text { intrauretric marcaine, }\end{array}$ & \\
\hline $\begin{array}{l}\text { Case } 7 \\
\text { Procedure } 6\end{array}$ & Stone & $\begin{array}{l}\text { Lower and } \\
\text { mid pole }\end{array}$ & & & $\begin{array}{l}\text { Paracetamol, } \\
\text { midazolam, } \\
\text { intrauretric marcaine, } \\
\text { propofol }\end{array}$ & \\
\hline $\begin{array}{l}\text { Case } 7 \\
\text { Procedure } 7\end{array}$ & Stone & Staghorn & & & $\begin{array}{l}\text { Paracetamol, } \\
\text { midazolam, } \\
\text { intrauretric marcaine, }\end{array}$ & \\
\hline $\begin{array}{l}\text { Case } 7 \\
\text { Procedure } 8\end{array}$ & Stone & Staghorn & & & $\begin{array}{l}\text { Paracetamol, } \\
\text { intrauretric marcaine }\end{array}$ & \\
\hline $\begin{array}{l}\text { Case } 7 \\
\text { Procedure } 9\end{array}$ & Stone & Staghorn & & & $\begin{array}{l}\text { Paracetamol, } \\
\text { intrauretric marcaine }\end{array}$ & \\
\hline $\begin{array}{l}\text { Case } 8 \\
\text { Procedure } 1\end{array}$ & Stone & Lower pole & $\begin{array}{l}\text { Partial } \\
\text { staghorn }\end{array}$ & & $\begin{array}{l}\text { Paracetamol, } \\
\text { midazolam, } \\
\text { intrauretric marcaine, } \\
\text { fentanyl, propofol }\end{array}$ & \multirow{4}{*}{$\begin{array}{l}\text { Failed ESWL. } \\
\text { Obstructive sleep } \\
\text { apnoea, chronic } \\
\text { obstructive } \\
\text { pulmonary disease }\end{array}$} \\
\hline $\begin{array}{l}\text { Case } 8 \\
\text { Procedure } 2\end{array}$ & Stone & Lower pole & $\begin{array}{l}\text { Partial } \\
\text { staghorn }\end{array}$ & DUR-D & $\begin{array}{l}\text { Paracetamol, } \\
\text { midazolam, } \\
\text { intrauretric marcaine }\end{array}$ & \\
\hline $\begin{array}{l}\text { Case } 8 \\
\text { Procedure } 3\end{array}$ & Stone & Lower pole & $\begin{array}{l}\text { Partial } \\
\text { staghorn }\end{array}$ & DUR-D & $\begin{array}{l}\text { Paracetamol, } \\
\text { midazolam, } \\
\text { intrauretric marcaine }\end{array}$ & \\
\hline $\begin{array}{l}\text { Case } 8 \\
\text { Procedure } 4\end{array}$ & Stone & Lower pole & Fragments & DUR-D & $\begin{array}{l}\text { Paracetamol, } \\
\text { midazolam, } \\
\text { intrauretric marcaine, } \\
\text { fentanyl, propofol }\end{array}$ & \\
\hline
\end{tabular}


TABLE 2 Current Case Series (Continued)

\begin{tabular}{|c|c|c|c|c|c|c|}
\hline $\begin{array}{l}\text { Case } 9 \\
\text { Procedure } 1\end{array}$ & Stone & & $\begin{array}{l}\text { Staghorn } \\
\text { (R) }\end{array}$ & URF-V & $\begin{array}{l}\text { Paracetamol, } \\
\text { midazolam, } \\
\text { intrauretric marcaine }\end{array}$ & $\begin{array}{l}\text { Spina bifida, patient } \\
\text { choice }\end{array}$ \\
\hline $\begin{array}{l}\text { Case } 9 \\
\text { Procedure } 2\end{array}$ & Stone & & $\begin{array}{l}\text { Staghorn } \\
\text { (R) }\end{array}$ & DUR-D & $\begin{array}{l}\text { Paracetamol, } \\
\text { midazolam, } \\
\text { intrauretric marcaine }\end{array}$ & \\
\hline $\begin{array}{l}\text { Case } 9 \\
\text { Procedure } 3\end{array}$ & Stone & & $\begin{array}{l}\text { Staghorn } \\
\text { (R) }\end{array}$ & DUR-D & $\begin{array}{l}\text { Paracetamol, } \\
\text { midazolam, } \\
\text { intrauretric marcaine }\end{array}$ & \\
\hline $\begin{array}{l}\text { Case } 9 \\
\text { Procedure } 4\end{array}$ & Stone & Lower pole & $12 \mathrm{~mm}$ & & $\begin{array}{l}\text { Paracetamol, } \\
\text { intrauretric Marcaine }\end{array}$ & \\
\hline $\begin{array}{l}\text { Case } 9 \\
\text { Procedure } 5\end{array}$ & Stone & Lower pole & $16 \mathrm{~mm}$ & & $\begin{array}{l}\text { Paracetamol, } \\
\text { intrauretric Marcaine }\end{array}$ & \\
\hline $\begin{array}{l}\text { Case } 9 \\
\text { Procedure } 6\end{array}$ & Stone & Lower pole & $16 \mathrm{~mm}$ & & $\begin{array}{l}\text { Paracetamol, } \\
\text { intrauretric marcaine }\end{array}$ & \\
\hline $\begin{array}{l}\text { Case } 9 \\
\text { Procedure } 7\end{array}$ & Stone & $\begin{array}{l}\text { Upper and } \\
\text { lower pole }\end{array}$ & & & $\begin{array}{l}\text { Paracetamol, } \\
\text { intrauretric marcaine }\end{array}$ & \\
\hline $\begin{array}{l}\text { Case } 9 \\
\text { Procedure } 8\end{array}$ & Stone & Lower pole & $15-20 \mathrm{~mm}$ & & $\begin{array}{l}\text { Paracetamol, } \\
\text { midazolam, fentanyl, } \\
\text { intrauretric marcaine }\end{array}$ & \\
\hline $\begin{array}{l}\text { Case } 9 \\
\text { Procedure } 9\end{array}$ & Stone & $\begin{array}{l}\text { Renal pelvis } \\
\text { and calyces }\end{array}$ & $\begin{array}{l}\text { Matrix } \\
\text { stone }\end{array}$ & & $\begin{array}{l}\text { Paracetamol, } \\
\text { midazolam, fentanyl, } \\
\text { intrauretric marcaine }\end{array}$ & \\
\hline $\begin{array}{l}\text { Case } 9 \\
\text { Procedure } \\
10\end{array}$ & Stone & Interpolar & $7-8 \mathrm{~mm}$ & & $\begin{array}{l}\text { Paracetamol, } \\
\text { midazolam, fentanyl, } \\
\text { intrauretric marcaine }\end{array}$ & \\
\hline Case 10 & Stone & Distal ureter & $7 \mathrm{~mm}$ & $\begin{array}{l}\text { Rigid } \\
\text { Scope }\end{array}$ & $\begin{array}{l}\text { Paracetamol, } \\
\text { midazolam, fentanyl }\end{array}$ & $\begin{array}{l}\text { Chronic obstructive } \\
\text { pulmonary disease, } \\
\text { Ischaemic heart } \\
\text { disease, spinal } \\
\text { deformity }\end{array}$ \\
\hline Case 11 & Stone & Mid ureter & $\begin{array}{l}18 \times 10 \\
\mathrm{~mm}\end{array}$ & $\begin{array}{l}\text { Rigid } \\
\text { Scope }\end{array}$ & $\begin{array}{l}\text { Paracetamol, } \\
\text { midazolam, propofol, } \\
\text { intrauretric marcaine }\end{array}$ & $\begin{array}{l}\text { Ischaemic heart } \\
\text { disease, coronary } \\
\text { artery bypass graft, } \\
\text { pulmonary fibrosis, } \\
\text { pleural plaques } \\
\text { (previous asbestos } \\
\text { exposure) }\end{array}$ \\
\hline
\end{tabular}


TABLE 2 Current Case Series (Continued)

\begin{tabular}{|l|l|l|l|l|l|l|}
\hline $\begin{array}{l}\text { Case 12 } \\
\text { Procedure 1 }\end{array}$ & Stone & $\begin{array}{l}\text { PUJ/ureteric } \\
\text { stone+ upper } \\
\text { pole }\end{array}$ & $7-12 \mathrm{~mm}$ & $\begin{array}{l}\text { Rigid } \\
\text { Scope }\end{array}$ & $\begin{array}{l}\text { Paracetamol, } \\
\text { midazolam, } \\
\text { intrauretric marcaine }\end{array}$ & $\begin{array}{l}\text { High body mass } \\
\text { index, warfarinised } \\
\text { for atrial fibrillation, } \\
\text { heart failure }\end{array}$ \\
\hline $\begin{array}{l}\text { Case 12 } \\
\text { Procedure 2 }\end{array}$ & Stone & $\begin{array}{l}\text { PUJ/ureteric } \\
\text { stone+upper } \\
\text { pole }\end{array}$ & $12-17 \mathrm{~mm}$ & $\begin{array}{l}\text { Rigid } \\
\text { Scope } \\
\text { pulmonary } \\
\text { hypertension, }\end{array}$ & $\begin{array}{l}\text { Paracetamol, } \\
\text { midazolam, } \\
\text { intrauretric marcaine } \\
\text { asthma }\end{array}$ \\
\hline $\begin{array}{l}\text { Case 12 } \\
\text { Procedure 3 }\end{array}$ & Stone & $\begin{array}{l}\text { PUJ/ureteric } \\
\text { stone+ upper } \\
\text { pole }\end{array}$ & $\begin{array}{l}12 \mathrm{~mm} \\
\text { residual } \\
\text { stone }\end{array}$ & $\begin{array}{l}\text { Rigid } \\
\text { Scope/ } \\
\text { DUR-D }\end{array}$ & $\begin{array}{l}\text { Paracetamol, } \\
\text { midazolam, } \\
\text { intrauretric marcaine }\end{array}$ & \\
\hline $\begin{array}{l}\text { Case 12 } \\
\text { Procedure 4 }\end{array}$ & Stone & $\begin{array}{l}\text { PUJ/ureteric } \\
\text { stone+ upper } \\
\text { pole }\end{array}$ & $\begin{array}{l}\text { Residual } \\
\text { stone }\end{array}$ & DUR-D & $\begin{array}{l}\text { Paracetamol, } \\
\text { midazolam, } \\
\text { intrauretric marcaine }\end{array}$ & \\
\hline
\end{tabular}

renal pathology a $35 \mathrm{~cm}$ or $45 \mathrm{~cm} \mathrm{12/14Fr} \mathrm{access}$ sheath was introduced into the ureter over the in-situ Sensor ${ }^{\circledR}$ wire and FURS was performed using the Olympus ${ }^{\circledR}$ URF-P5, Olympus ${ }^{\circledR}$ URF-V and GyrusACMI DUR-D endoscopes. Laser fragmentation of stones or ablation of carcinoma was performed using a Holmium: YAG laser all the while with close monitoring of patients both physiologically and clinically. A double-J stent was inserted at the end of selected procedures under image intensifier guidance either on a string for removal in three days or by flexible cystoscopic removal in due course.

\section{DATA COLLECTION}

Data was collected retrospectively from patient notes and by reviewing radiological imaging. The indication for (F)URS and for avoiding general and spinal anesthetic was recorded. The site of pathology and stone burden was determined from operation notes and imaging. In cases where no recent imaging was available to determine stone size, this data was left missing (see Table 2). The use of sedation and analgesia was identified from the anesthetic and drug charts.

\section{ETHICS}

Informed consent was gained for all procedures. No further ethics approval was required as operations were performed as the standard of care and this was an audit of existing practise.

\section{THE RESULTS OF THE CASE SERIES}

Twelve patients had a total of 42 procedures under LA over six years from 2010 to 2016. Stone size varied from 4-35mm. Twenty-two percent of procedures (9/41) did not require any sedation or intravenous analgesia as an adjunct to the per-urethral instillagel ${ }^{\circledR}$ or intra-ureteric marcaine. The anesthetic chart could not be located for one procedure. No procedures were abandoned due to pain and there were no conversions to GA. There were no complications secondary to LA. Eighty-one percent of cases (34/42) were performed as day case or overnight stays. The overall complication rate was $14 \%$.

\section{THE RESULTS OF THE SYSTEMATIC REVIEW}

The search process yielded 45 possible articles of which 34 were deemed not suitable for inclusion as the data was not available or not clear after abstract screening. In total, 11 papers generated from 7 countries, with a total of 1121 procedures were included. The mean age from the collective group was 44 years-old and the male to female ratio 0.92:1. All included studies were single centre. Eight articles were prospective, ${ }^{5-14}$ one of which was a randomized controlled trial. ${ }^{4}$ Two were retrospective studies. ${ }^{7,15}$

\section{OUTCOME MEASURES}

\section{Safety}

Out of a total 1121 procedures, 32 procedures were converted to general anesthesia (GA), mostly 
for pain and inability to tolerate the procedure and also the need for a prolonged procedure. Postoperative pain was reported in 10 patients and 19 patients had a ureteric laceration or injury to the urinary tract. Two patients had false passages, two patients had ureteric strictures and nine developed postoperative urinary tract infections either cystitis or pyelonephritis

\section{Stone Clearance Rate}

In total, more than 861 patients had URS for ureteric calculi, achieving a stone-free rate of between $78 \%$ and $100 \%$. This is comparable to procedures undertaken under conventional anesthesia.

\section{Patient Tolerability}

Few papers in this review commented on the tolerability of the procedure under LA or with some degree of sedation. Rittenberg et al. reported on the procedure being well tolerated in all patients. ${ }^{5}$ Yalcincaya et al. commented on mild pain in $4 / 30$, moderate pain in $5 / 30$ of patients and intolerable pain in $2 / 30 .{ }^{4}$ Hosking et al. reported good tolerance in $81.4 \%$ and fair tolerance in $18.6 \% .{ }^{6}$ Park et al. reported a mean pain score of 3.4 (on 10 -point scale). ${ }^{7}$ Rao et al. reported $79 \%$ of participants having mild pain with only $10 \%$ having severe pain. ${ }^{8}$

\section{Length of Hospital Stay}

Only one paper commented on length of stay as an outcome measure ${ }^{7}$ with mean length of stay between 0.58 and 0.96 days for patients having URS with a combination of diclofenac, promethazine, metoclopramide, midazolam and urethral lidocaine.

\section{Discussion}

This review has demonstrated that although not a popular technique, ureteroscopy under sedoanalgesia is viable and safe. This corroborates the finding of our own series whereby in high-risk surgical candidates this approach can be a useful tool. The patients in our series demonstrate this with three of the 12 having had previous intensive care unit (ITU) admissions following GA and a further five deemed at very high risk of ITU admission or death if they were to be given a GA. For this reason, operating under LA is advantageous, if not essential, for a number of patients.

Our systematic review has shown that FURS has been described with intra-urethral LA and sedation. This has been shown to be well tolerated and successful. ${ }^{4-14}$
There are, however, no reports of the use of intraureteric LA as an adjunct to this, which has allowed the treatment of larger renal stones including staghorn calculi through a multi-phased approach in our series. The treatment of such stones has not been described with intra-urethral LA, sedation and analgesia alone.

Often, the arguments against the use of LA are the perceived poor patient tolerability and risk of ureteric injury from patient movement. However, two studies have compared subjective pain scores of various urological procedures, including rigid cystoscopy, ESWL and FURS and not shown FURS to be more painful. ${ }^{7,12}$ In our series of 42 procedures there were no abandoned procedures due to pain. Furthermore, $43 \%$ of procedures were successfully performed with only paracetamol and/ or a non-steroidal antiinflammatory drug (NSAID) as an adjunct to the LA. In six procedures, a dose of propofol was required to maintain anesthesia. There was one case of ureteric perforation in our cohort, which resulted from damage caused by the guide wire. This was not associated with any patient movement and cannot be attributed to LA. This patient was successfully managed conservatively with a double-J stent and on subsequent imaging and follow up was not found to have hydronephrosis or impaired drainage on the affected side.

Rittenberg et al. were the first to report ureteroscopy under LA. ${ }^{5}$ In this cohort of 30 patients, 18 patients had flexible ureterorenoscopy, seven patients had rigid ureteroscopy, and five had both. Of these procedures, 25 ureteroscopies were performed for diagnostic purposes and five for ureteric calculi; in one case for a mid-ureteric stone, and four were in the distal ureter. All the procedures were performed using $2 \%$ lidocaine per urethra. Twenty-eight out of 30 patients had intravenous midazolam in addition. There were no reported complications and stone clearance was found to be $100 \%$.

Chan et al. demonstrated the feasibility of transurethral ureteroscopic lithotripsy under LA and sedation, in a prospective study of 61 patients using $10 \mathrm{mg}$ of diazepam orally. ${ }^{8}$ The stone clearance rate was $78 \%$ with no major complications reported and one conversion to GA to achieve stone clearance. This study also demonstrated reasonable tolerance to the procedure with a mean pain score of 6 on a pain scale 
of 0 to $10(0=$ no pain; $10=$ extreme unbearable pain $)$. Importantly, it showed no difference in the duration of treatment between successful and unsuccessful procedures.

In 1993 Vogeli et al., in their prospective study, reported on 133 patients who successfully completed ureteroscopic procedures without the need for general or regional anesthesia from a total of 161 patients. ${ }^{9}$ Interestingly, the study highlighted that time required to perform the treatment was a major factor, unlike the findings of Chan et al. In the same year, AbdelRazzak et al. performed a retrospective study using $6.9 \mathrm{~F}$ ureteroscopes in 65 procedures in 57 patients. ${ }^{10}$ Out of these, 42 patients were treated with intraurethral topical anesthesia and intravenous sedation.

In 1995, Yalcinkaya et al. conducted a prospective randomized study of 35 patients divided into two groups depending on stone position. ${ }^{4}$ The study concluded that there was no significant difference in the stone-free rate of ureteroscopy when comparing cases performed under GA versus those under LA with sedation. Similarly, there were no additional major complications. Just one year later, Hosking et al. reported the results of 70 patients' data that underwent ureteroscopy with intravenous sedation. ${ }^{6}$ They reported a stone clearance rate of $95.7 \%$ in 67 patients and failed procedures in three patients due to a larger stone burden and needing manipulation under GA. This study highlighted the overall tolerance as "good" in $81.4 \%$ and "fair" in $18.6 \%$ of patients. Miroglu et al. in 1997 reported 51 procedures under LA and intravenous analgesia for varying pathologies. ${ }^{11}$ This study presented 34 procedures performed for ureteric stones and reported stone clearance in $83 \%$.

Park et al. in 2004 reported their retrospective series of 200 cases of LA ureteroscopic procedures, primarily performed for ureteric calculi. The authors compared pain during cystoscopy and LA ureteroscopic lithotripsy using a visual analog scale in a total of 55 patients who underwent LA ureteroscopic lithotripsy and 226 patients who underwent cystoscopy. This study reported an overall stone-free rate of $93 \%$ $(185 / 200)$. One patient had a ureteric injury, two had pyelonephritis and two had ureteric strictures. The mean pain score after LA ureteroscopic lithotripsy was 3.4, compared with 3.1 after cystoscopy alone.
The difference between these groups was reported as being statistically insignificant.

Jeong et al. in 2005, investigated subjective pain generated during extra-corporeal shockwave lithotripsy (ESWL) and endoscopic procedures such as ureteroscopic lithotripsy, cystoscopy, retrograde ureteric stenting and retrograde pyelography (RGP) performed as a day case procedure. ${ }^{12}$ In this prospective study, subjective pain was evaluated using a 10-point linear visual analog scale (VAS). They concluded that ureteroscopic lithotripsy along with other endoscopic procedures had a pain score significantly lower than ESWL which is traditionally performed with relatively mild analgesia and rarely sedation. In a prospective study of 124 patients with ureteric calculi, Rao et al. reported on ureteroscopic lithotripsy under sedoanalgesia with the use of $2 \%$ lidocaine as LA. ${ }^{12}$ The authors concluded that the procedure was well tolerated by patients with no reported complications or technical difficulties. In two patients, the procedure was abandoned because of intolerable pain. The patients with the worst pain in both the assessments had mid-ureteric calculi.

In 2006, Gupta et al. described treating patients with ureteric calculi with LA alone or with sedation. Several patients had "monitored anesthesia" with propofol or ketamine. ${ }^{14}$ No complications during or after the procedure were reported, with no patients had postoperative pain. The author reports stone clearance in 23 out of 25 patients.

Recently, one of the biggest retrospective studies of 10 years by Kroczak et al. reviewed data from 314 ureteroscopic procedures performed for distal ureteric calculi. ${ }^{15}$ The mean age of patients in this cohort was 53.7 years, with 160 males and 154 females. Procedures were performed with initial doses of $100 \mu \mathrm{g}$ of fentanyl and $2 \mathrm{mg}$ of midazolam administered prior to the start of the procedure. An additional dose of fentanyl was given in 25-50 $\mu \mathrm{g}$ increments and midazolam in $1 \mathrm{mg}$ doses. The author reported a success rate of $97 \%$ and a $3.2 \%$ complication rate. A total of 263 patients $(83.7 \%)$ tolerated the procedure well, with seven $(2.2 \%)$ having poor tolerability.

The differences in the varying complication and success rates could be explained in part by the considerable variants in endourological equipment with 
older series using larger calibre scopes. The oldest papers in the review are over 30 years-old and this may be responsible for differences in results between series. Table 3 displays the different complications noted in the systematic review.

In our series, flexible ureteroscopy was been performed using intra-ureteric marcaine as the primary form of anesthesia. Using intra-ureteric marcaine $(0.5 \%, 20$ $\mathrm{mL}$ ) via a ureteric catheter, 12 patients had a total of 42 procedures under LA. Twenty-two percent of these did not require any sedation or intravenous analgesia as an adjunct to the marcaine. Table 4 displays the sedoanalgesia used. No procedures were abandoned due to pain, there were no conversions to GA and no complications secondary to LA. In one patient, who had pain every time the pathfinder irrigation bulb was compressed by the surgeon had a further $20 \mathrm{~mL}$ $0.25 \%$ marcaine infused under pressure into the renal pelvis. Within a few minutes the patient was pain free with irrigation.

Four of our patients had staghorn/ partial staghorn stones. Three patients had large renal stone burdens and in two, additional ureteric and renal stones, four had ureteric stones only and one had ureteric TCC. One patient's operative time was limited to 30 minutes due to a previous episode of decompensated heart failure precipitated by anesthesia. At the follow-up procedure, they developed pyelonephritis accompanied by pain and were managed conservatively. Three other procedures resulted in postoperative pyrexia which was again managed conservatively with intravenous antibiotics and fluid resuscitation. These patients had large stone burdens with all having (partial) staghorn stones. One patient developed a perinephric abscess, presenting four weeks after their fourth and final procedure for an initial large 35-mm staghorn calculus and had this drained percutaneously by the radiologist making a good recovery clinically and radiologically.

One patient with a $10-\mathrm{mm}$ upper-ureteric stone and a 4-mm vesico-ureteric junction stone on the ipsilateral side had a guide-wire related perforation of the ureter which was recognized intra-operatively and managed with a double-J stent left in-situ for four weeks post-operatively. It was not thought to be due to inadequate analgesia resulting in a sudden movement of the patient but would likely have occurred under GA in the same way.

One of our patients with TCC in their distal ureter tolerated the procedure poorly, but to completion allowing laser ablation of the tumour. Subsequent

TABLE 3 Complications Noted in the Systematic Review

\begin{tabular}{|l|c|}
\hline Complications & Number of Cases \\
\hline Converted to general anesthesia & 32 \\
\hline Ureteric laceration / injury & 19 \\
\hline Ureteric false passage & 2 \\
\hline Ureteric strictures & 2 \\
\hline Urinary sepsis (pyelonepritis/cystitis) & 9 \\
\hline Post-operative pain & 10 \\
\hline
\end{tabular}

TABLE 4 Type of Sedoanalgesia Used in Current Series. (Case 5 Procedure 4 Lacked Anesthetic Chart)

\begin{tabular}{|l|c|}
\hline Sedoanalgesia Used & Number of Cases \\
\hline A: Marcaine +/- paracetmol +/- NSAID & 9 \\
\hline B: A + midazolam & 20 \\
\hline C: B + propofol +/- fentanyl & 12 \\
\hline
\end{tabular}

J Endolum Endourol Vol 2(2):e1-e14; May 1, 2019.

This article is distributed under the terms of the Creative Commons Attribution-Non

Commercial 4.0 International License. (CTasleem et al. 
procedures were therefore performed under GA, as the choice here initially was the patient's to attempt the procedure under LA.

One of our patients had 10 procedures and continues to have planned maintenance ureteroscopies due to her history of an initial large staghorn stone with recurrent stone formation and large amounts of matrix stone between procedures. After an initial period of conservative management, she developed infections requiring antibiotics. Periodic ureteroscopies with washouts of matrix material and stone fragmentation was found to reduce the frequency of infections and was the strategy adopted moving forward. There was another patient with a large stone burden who required multiple procedures and therefore this increases the mean number of procedures per patient overall in our cohort to 3.5 which is higher than the usual re-look rate.

The majority $(81 \%, 34 / 42)$ of our patients were discharged in less than 24 hours of their procedure. The longest stay was 6 days in the patient who developed a perinephric abscess. See Table 5 for the length of stay.

From our experience, we found some key aspects were important in order to reduce the pain and to improve tolerance. Initial counselling with a full and comprehensive description of the procedure in clinic is paramount to the patient's perception of what they are due to undergo with the provision of British Association of Urological Surgeons (BAUS) information leaflets. We believe time and effort spent explaining details to the patient affected how they went on to tolerate surgery moving forward. The operating surgeon saw the patients in clinic himself, on the morning of surgery and post-operatively. Intra-operatively, the bladder was not allowed to over-distend and the patient's voluntary urination alongside the scope, which was encouraged, helped keep the bladder empty. Distension of the

TABLE 5 Length of Stay in Current Series

\begin{tabular}{|l|c|}
\hline \multicolumn{1}{|c|}{ Length of Stay } & Number of Cases \\
\hline $\begin{array}{l}\text { Daycase (admitted and } \\
\text { discharged same day) }\end{array}$ & 12 \\
\hline$<24 \mathrm{hr}$ & 22 \\
\hline $48 \mathrm{hr}$ & 4 \\
\hline$>48 \mathrm{hr}$ ( Range 3-6 days) & 4 \\
\hline
\end{tabular}

renal pelvis was kept to minimum using a pathfinder irrigation bulb allowing the surgeon to control flow rate tightly. We also noted that an important part of tolerability for patients was talking to them throughout the procedure, explaining what was going on and reassuring them. This was often achieved by showing them the surgery live on a slave-monitor adjacent to them and a member of theatre staff being present next to the patient at all times.

Intra-ureteric administration of marcaine, a long acting LA, has been previously reported in patients with chronic renal pain ${ }^{16}$; however, as far as we are aware this is the first report of its use as an adjunct to ureteroscopy.

Our study has limitations including absence of a control group of patients not treated with marcaine and also an objective assessment of patient's pain. However, according to the analysis of notes and from the team's experience; other than the one patient mentioned in the current series, the rest of our cohort tolerated these procedures well. The small number of patients involved also limits generalization. Conversely, the success rate reported in the literature and large number of patients who may potentially benefit warrants further investigation.

\section{CONCLUSION}

This review article shows that URS and FURS can be safely performed under LA with or without sedation and intravenous analgesia when indicated. It is largely well tolerated and avoids the risks associated with general and spinal anesthesia in the unfit patient that might otherwise not be considered for such endourological procedures. This option is useful in those patients who may not want general or spinal anesthesia and also in those whereby a LA percutaneous nephrolithotomy may be contraindicated or high risk. Further prospective trials are required to investigate efficacy, success rates and ascertain specific criteria for its use.

\section{REFERENCES}

1. Wills TE, Burns JR. Ureteroscopy: an outpatient procedure? J Urology 1994;151:1185-7.

2. Goodman TM. Ureteroscopy with paediatric cystoscope in adults. Urology 1977;9:394. 
3. Aboumarzouk, Omar M, Bhaskar K. Somani, and Manoj Monga. Flexible ureteroscopy and holmium:yag laser lithotripsy for stone disease in patients with bleeding diathesis: a systematic review of the literature. Int Braz J Urol 2012;38.3:298-306.

4. Yalcinkaya F, Topaloglu H, Ozmen E, Unal S. Is general anesthesia necessary for URS in women? Int Urol Nephrol 1996;28.2:153-56.

5. Rittenberg, Michael H., David J. Ellis, and Demetrius H. Bagley. Ureteroscopy under local anesthesia. Urology 1987;30.5:475-78.

6. Hosking DH, Bard RJ. Ureteroscopy with intravenous sedation for treatment of distal ureteral calculi: a safe and effective alternative to shock wave lithotripsy. J Urol 1996;Sep;156(3):899-901; discussion 902.

7. Park, Hyoung Keun et al. Ureteroscopic Lithotripsy under local anesthesia: analysis of the effectiveness and patient tolerability. Eur Urol 2004;45.5:670-73.

8. Chan PSF, Fenn J. and LI AKC. Transurethral Ureterorenoscopic Lithotripsy and retrieval of ureteric calculi under local anesthesia and sedation. Br J Urol 1990;65:141-43.

9. Vogeli T-A. et al. Ureteroscopy under local anesthesia with and without intravenous analgesia. Br J Urol $1993 ; 72.2: 161-64$
10. Abdel-Razzak $\mathrm{O}$ and Bagley DH. The $6.9 \mathrm{~F}$ semi-rigid ureteroscope in clinical use. Urolog 1993;41(1):45-48.

11. Miroglu C, Saporta L. Transurethral ureteroscopy: is local anesthesia with intravenous sedation sufficiently effective and safe? Eur Urol 1997;31:36-39

12. Jeong B, Park C, Kwak H. et al. How painful are shockwave lithotripsy and endoscopic procedures performed at outpatient urology clinics? Urological Res 2005;33(4):291-96.

13. Rao MP, Kumar S, Dutta B, et al. Safety and efficacy of ureteroscopic lithotripsy for ureteral calculi under sedoanalgesia - a prospective study. Int Urol Nephrol 2005;37.2:219-24.

14. Gupta PK. Initial experience with a prototype ureteroscope. J Endourol 2006;20.1:9-11.

15. Kroczak TJ, Kamaljot SK, Patel P, and Al-Essawi T. Ureteroscopy with conscious sedation for distal ureteric calculi: 10-year experience. Can Urolog Assoc J. 2016;10(1 2):E12.

16. Ahmed P, Acher P, and Deane A. Ureteric bupivacaine infusion for loin pain haematuria syndrome. Ann Royal Coll Surg Eng 2010;92(2):139-41. 